\title{
Study on the numerical model of dense solid suspension driven by
}

\section{coaxial mixer}

Zilong Xu, Chao Yang, Ziqiang Zhang, Baoqing Liu*, Zhijiang Jin*

(Institute of Process Equipment, Zhejiang University, Hangzhou 310027, China)

* To whom correspondence should be addressed

E-mail address: baoqingliu@ zju.edu.cn, zhijiangjin@126.com 
Table S1. Studies of coaxial mixers

\begin{tabular}{|c|c|c|c|c|}
\hline Source & Configuration & Method & Fluid type & Parameter \\
\hline Liu et $\mathrm{al}^{21}$ & $\begin{array}{l}\text { Rushton and pitched } \\
\text { blade-Anchor } \\
\text { Rushton and straight } \\
\text { blade-Anchor }\end{array}$ & $\begin{array}{l}\text { Experimental } \\
\text { method }\end{array}$ & Newtonian fluid & Segregation index \\
\hline Kazemzadeh et al. ${ }^{22}$ & $\begin{array}{l}\text { Two central Scaba } \\
\text {-Anchor }\end{array}$ & $\begin{array}{l}\text { Experimental and } \\
\text { numerical methods }\end{array}$ & non-Newtonian fluid & $\begin{array}{c}\text { Power number } \\
\text { Flow number } \\
\text { Mixing time }\end{array}$ \\
\hline Liu et al. ${ }^{23}$ & $\begin{array}{l}\text { Rushton-Anchor } \\
\text { Straight blade-Anchor } \\
\text { Pitched blade-Anchor }\end{array}$ & $\begin{array}{l}\text { Experimental } \\
\text { method }\end{array}$ & Newtonian fluid & Power consumption \\
\hline Farhat et al. ${ }^{24}$ & Rushton-Anchor & $\begin{array}{l}\text { Experimental } \\
\text { method }\end{array}$ & Newtonian fluid & $\begin{array}{c}\text { Power consumption } \\
\text { Mixing time }\end{array}$ \\
\hline Hashemi et al. ${ }^{25}$ & Pitched blade-Anchor & Numerical method & Gas-liquid & $\begin{array}{c}\text { Gas holdup } \\
\text { flow pattern } \\
\text { turbulent kinetic energy }\end{array}$ \\
\hline Jegatheeswaran et al. ${ }^{26}$ & $\begin{array}{l}\text { Lightnin A200-Anchor } \\
\text { Lightnin A315-Anchor } \\
\text { Lightnin A320-Anchor }\end{array}$ & $\begin{array}{l}\text { Experimental and } \\
\text { numerical methods }\end{array}$ & Gas-liquid & $\begin{array}{c}\text { Gas holdup } \\
\text { Gas velocity } \\
\text { Power consumption }\end{array}$ \\
\hline $\begin{array}{c}\text { Jegatheeswaran and } \\
\text { Ein-mozaffari }^{27}\end{array}$ & Scaba-Anchor & $\begin{array}{l}\text { Experimental and } \\
\text { numerical methods }\end{array}$ & Gas-liquid & $\begin{array}{c}\text { Gas holdup } \\
\text { Flow number } \\
\text { Power consumption }\end{array}$ \\
\hline Jamshidzadeh et al. $^{28}$ & $\begin{array}{c}\text { Scaba and pitched blade } \\
\text {-Anchor }\end{array}$ & $\begin{array}{l}\text { Experimental and } \\
\text { numerical methods }\end{array}$ & Gas-liquid & Gas holdup \\
\hline Jamshidzadeh et al. $^{29}$ & $\begin{array}{l}\text { Two central pitched } \\
\text { blade-Anchor }\end{array}$ & $\begin{array}{l}\text { Experimental and } \\
\text { numerical methods }\end{array}$ & Gas-liquid & Power consumption \\
\hline Liu et al. ${ }^{30}$ & $\begin{array}{c}\text { Rushton-Anchor } \\
\text { Propeller-Anchor } \\
\text { Pitched blade-Anchor }\end{array}$ & $\begin{array}{c}\text { Experimental } \\
\text { method }\end{array}$ & Solid-liquid & $\begin{array}{l}\text { Just-suspended speed } \\
\text { Just-suspended power }\end{array}$ \\
\hline $\mathrm{Xu}$ et al. ${ }^{31}$ & $\begin{array}{l}\text { Rushton-Anchor } \\
\text { Propeller-Anchor }\end{array}$ & $\begin{array}{l}\text { Experimental } \\
\text { method }\end{array}$ & Solid-liquid & $\begin{array}{l}\text { Just-suspended power } \\
\text { Cloud height }\end{array}$ \\
\hline Liu et al. ${ }^{32}$ & Rushton-Anchor & Numerical method & Solid-liquid & $\begin{array}{l}\text { Solid volume fraction } \\
\text { Solid velocity } \\
\text { Cloud height }\end{array}$ \\
\hline
\end{tabular}


Table S2. Equations of drag models

Model Equation

$\mathbf{F}_{s l}=-\mathbf{F}_{l s}=K_{s l}\left(\mathbf{v}_{s}-\mathbf{v}_{l}\right)$

Gidaspow model: $\quad K_{s l}=150 \frac{\alpha_{s}\left(1-\alpha_{l}\right) \mu_{l}}{\alpha_{l} d_{s}^{2}}+1.75 \frac{\rho_{l} \alpha_{s}\left|\mathbf{v}_{s}-\mathbf{v}_{l}\right|}{d_{s}} \quad\left(\alpha_{l} \leq 0.8\right)$

$K_{s l}=\frac{3}{4} C_{D} \frac{\alpha_{s} \alpha_{l} \rho_{l}\left|\mathbf{v}_{s}-\mathbf{v}_{l}\right|}{d_{s}} \alpha_{l}^{-2.65} \quad\left(\alpha_{l}>0.8\right)$

where $C_{D}=\frac{24}{\alpha_{l} \operatorname{Re}_{s}}\left[1+0.15\left(\alpha_{l} \operatorname{Re}_{s}\right)^{0.687}\right]$

Brucato model: $\quad K_{s l}=\frac{3 \alpha_{s} \rho_{l}}{4 d_{s}} C_{D}\left|\mathbf{v}_{s}-\mathbf{v}_{l}\right|$

$C_{D}=C_{D o}\left[1+8.76 \times 10^{-4}\left(\frac{d_{s}}{\lambda}\right)^{3}\right]$

$C_{D o}=\frac{24}{R e_{s}} \times\left(1+0.15 R e_{s}^{0.687}\right)$

Clift model: $\quad K_{s l}=\frac{3 \alpha_{s} \rho_{l}}{4 d_{s}} C_{D}\left|\mathbf{v}_{s}-\mathbf{v}_{l}\right|$

where $C_{D}=\left\{\begin{array}{cc}\frac{24}{R e_{s}}\left(1+0.2 R e_{s}^{0.63}\right) & R e_{s} \leq 1000 \\ 0.44 & R e_{s}>1000\end{array}\right.$

Hill-Koch-Ladd model: $\quad K_{s l}=18 \mu_{l}\left(1-\alpha_{s}\right)^{2} \alpha_{s} \frac{F}{d_{s}^{2}}$

Where $F=1+\frac{3}{8} R e_{s}, \quad \alpha_{s} \leq 0.01$ and $R e_{s} \leq \frac{F_{2}-1}{(3 / 8)-F_{3}}$

$F=F_{0}+F_{1} R e_{s}^{2}, \quad \alpha_{s}>0.01$ and $R e_{s} \leq \frac{F_{3}+\sqrt{F_{3}^{2}-4 F_{1}\left(F_{0}-F_{2}\right)}}{2 F_{1}}$

$F=F_{2}+F_{3} R e_{s} \begin{cases}\alpha_{s} \leq 0.01 \quad \text { and } & R e_{s}>\frac{F_{2}-1}{(3 / 8)-F_{3}} \\ \alpha_{s}>0.01 & \text { and } \quad R e_{s}>\frac{F_{3}+\sqrt{F_{3}^{2}-4 F_{1}\left(F_{0}-F_{2}\right)}}{2 F_{1}}\end{cases}$ 


$$
\begin{aligned}
& F_{0}=\left\{\begin{array}{lc}
(1-w)\left[\frac{1+3 \sqrt{\alpha_{s} / 2}+135 \alpha_{s} \ln \left(\alpha_{s}\right) / 64+17.14 \alpha_{s}}{1+0.681 \alpha_{s}-8.4 \alpha_{s}{ }^{2}+8.16 \alpha_{s}{ }^{3}}\right]+w\left[\frac{10 \alpha_{s}}{\left(1-\alpha_{s}\right)^{3}}\right] & 0.01<\alpha_{s}<0.4 \\
\frac{10 \alpha_{s}}{\left(1-\alpha_{s}\right)^{3}} & \alpha_{s} \geq 0.4
\end{array}\right. \\
& F_{1}= \begin{cases}\frac{\sqrt{2 / \alpha_{s}}}{40} & 0.01<\alpha_{s} \leq 0.1 \\
0.11+0.00051 e^{11.6 \alpha_{s}} & \alpha_{s}>0.1\end{cases} \\
& F_{2}= \begin{cases}(1-w)\left[\frac{1+3 \sqrt{\alpha_{s} / 2}+135 \alpha_{s} \ln \left(\alpha_{s}\right) / 64+17.14 \alpha_{s}}{1+0.681 \alpha_{s}-8.4 \alpha_{s}^{2}+8.16 \alpha_{s}^{3}}\right]+w\left[\frac{10 \alpha_{s}}{\left(1-\alpha_{s}\right)^{3}}\right] & \alpha_{s}<0.4 \\
\frac{10 \alpha_{s}}{\left(1-\alpha_{s}\right)^{3}} & \alpha_{s} \geq 0.4\end{cases} \\
& F_{3}= \begin{cases}0.9351 \alpha_{s}+0.03667 & \alpha_{s}<0.0953 \\
0.0673+0.212 \alpha_{s}+\frac{0.0232}{\left(1-\alpha_{s}\right)^{5}} & \alpha_{s} \geq 0.0953\end{cases} \\
& R e_{s}=\frac{\rho_{l}\left(1-\alpha_{s}\right) d_{s}\left|\mathbf{v}_{s}-\mathbf{v}_{l}\right|}{2 \mu_{g}} \\
& w=e^{\left(-10\left(0.4-\alpha_{s}\right) / \alpha_{s}\right)}
\end{aligned}
$$

